\title{
Cardiovascular Health and Disease in the Context of COVID-19
}

\author{
Sidhant Umbrajkar ${ }^{\mathrm{a}, \mathrm{b}}$, Rachel V. Stankowskic, Shereif Rezkalla ${ }^{\mathrm{d}}$, \\ Robert A. Kloner ${ }^{\text {b, e, } f}$
}

\begin{abstract}
First documented in China in early December 2019, the novel severe acute respiratory syndrome coronavirus 2 (SARS-CoV-2) spread rapidly and continues to test the strength of healthcare systems and public health programs all over the world. Underlying cardiovascular disease has been recognized as a risk factor for coronavirus disease 2019 (COVID-19)-related morbidity and mortality since the early days of the pandemic. In addition, evidence demonstrates cardiac and endothelial damage in somewhere between one-third and three-quarters of individuals with COVID-19, regardless of symptom severity. This damage is thought to be mediated by direct viral infection, immunopathology and hypoxemia with the additional possibility of exacerbation via medication-induced cardiotoxicity. Clinically, the cardiovascular consequences of COVID-19 may present as myocarditis with or without arrhythmia, endothelial dysfunction and thrombosis, acute coronary syndromes and heart failure. Presentation can vary widely and may or may not be typical of the condition in an individual without COVID-19. There is evidence to support the prognostic utility of cardiac biomarkers (e.g., cardiac troponin) and imaging studies (e.g., echocardiography, cardiac magnetic resonance imaging) in the context of COVID-19 and building evidence suggests that cardiovascular screening may be warranted even among those with asymptomatic or mild infection and those without traditional cardiovascular risk factors. In addition, evidence suggests the potential for long-term cardiovascular consequences for those who recover from COVID-19 with implications for the field of cardiology long into the future. Even among those without COVID-19, disruption of infrastructure and
\end{abstract}

Manuscript submitted November 15, 2020, accepted December 17, 2020

Published online February 23, 2021

aUniversity of California, Los Angeles (UCLA), 10833 Le Conte Ave., Los Angeles, CA 90095, USA

bHuntington Medical Research Institutes, 686 South Fair Oaks Ave., Pasadena, CA 91105, USA

'Family Health Center of Marshfield, Inc., Marshfield Clinic Health System, 1000 N Oak Ave., Marshfield, WI 54449, USA

dDepartment of Cardiology, Marshfield Medical Center, 1000 N Oak Ave., Marshfield, WI 54449, USA

${ }^{e}$ Keck School of Medicine of the University of Southern California, 1975 Zonal Ave., Los Angeles, CA 90033, USA

${ }^{f}$ Corresponding Author: Robert Kloner, Cardiovascular Research Institute, Huntington Medical Research Institutes, 686 South Fair Oaks Ave., Pasadena, CA 91105, USA. Email: robert.kloner@hmri.org

doi: https://doi.org/10.14740/cr1199 changes in human behavior as a result of the pandemic also have an upstream role in cardiovascular outcomes, which have already been documented in multiple locations. This review summarizes what is currently known regarding the pathogenic mechanisms of COVID19-related cardiovascular injury and describes clinical cardiovascular presentations, prognostic indicators, recommendations for screening and treatment, and long-term cardiovascular consequences of infection. Ultimately, medical personnel must be vigilant in their attention to possible cardiovascular symptoms, take appropriate steps for clinical diagnosis and be prepared for long-term ramifications of myocardial injury sustained as a result of COVID-19.

Keywords: COVID-19; SARS-CoV-2; Cardiovascular disease; Myocardial damage; Endothelial dysfunction; Viral infection; Inflammation

\section{Introduction}

First documented in China in early December 2019, the novel severe acute respiratory syndrome coronavirus 2 (SARSCoV-2) spread rapidly and continues to test the strength of healthcare systems and public health programs all over the world. As of this writing, the United States has reached more than 10 million cases of coronavirus disease 2019 (COVID-19) and over 240,000 deaths. Worldwide there have been nearly 53 million cases and 1.3 million deaths. SARS-CoV-2 spreads primarily from human to human through respiratory droplets from coughing, sneezing, or talking; although aerosol transmission from very small particles and droplets that float in the air for minutes to hours is now thought to play a role [1]. Since the early days of the COVID-19 pandemic, underlying cardiovascular disease has been recognized as a risk factor for morbidity and mortality [2]. More recently, the cardiovascular consequences of COVID-19 infection, even among those who are young and otherwise healthy and those who have had no other symptoms, have caused concern [3].

The frequency of cardiovascular complications is thought to be relatively high among individuals with COVID-19, although reported rates of cardiac involvement vary widely among hospitalized patients as a result of different diagnostic modalities and inclusion and exclusion of patients with various underlying conditions. For example, the National Health Commission of China reports that $11.8 \%$ of patients without under- 
lying cardiovascular comorbidities had evidence of substantial heart damage during hospitalization [4]. On the opposite end of the spectrum, Szekely et al conducted a systematic echocardiographic study of 100 consecutive patients diagnosed with COVID-19 within $24 \mathrm{~h}$ of admission and found that $78 \%$ of patients had evidence of cardiac pathology [5]. Use of biomarkers, such as high-sensitivity troponin (hs-troponin) to detect myocardial injury has resulted in rates somewhere in between, with Arcari et al noting elevation in 38\% of 111 patients with COVID-19 admitted to a hospital in Italy [6], and Shah et al noting elevation in $37.5 \%$ of 309 patients with COVID-19 admitted to a hospital in the southern United States [7]. Rates of cardiac involvement are harder to estimate among individuals with COVID-19 not requiring hospitalization, although a report suggesting myocardial involvement in $46 \%$ of 26 competitive college athletes with mild or no symptoms suggests that it may be relatively common even among those who are otherwise young and healthy [8]. More strikingly, in an unselected sample of 100 individuals who recovered from COVID-19, Puntmann et al identified cardiac involvement in $78 \%$ with no correlation between cardiac involvement and severity or extent of disease [9]. Numerous studies agree that cardiovascular involvement is associated with poorer prognosis in the context of COVID-19, regardless of the frequency with which it occurs. Launched in April, the American Heart Association COVID-19 CVD Registry is poised to support rapid advancement in our understanding of the impact of COVID-19 on cardiovascular disease and how to improve management strategies [10]. As we await data accrual and analysis, this review summarizes what is currently known regarding the pathogenic mechanisms of COVID-19-related cardiovascular injury and describes clinical cardiovascular presentations, prognostic indicators, recommendations for screening and treatment, and long-term cardiovascular consequences of infection.

\section{Pathogenic Mechanisms of Cardiovascular Injury}

Numerous reports in the literature describe both cardiac and endothelial damage among those with COVID-19. Pathogenic mechanisms of cardiovascular damage include direct viral infection, immunopathology and hypoxemia with the additional possibility of exacerbation via medication-induced cardiotoxicity. Further, disruption of infrastructure and changes in human behavior as a result of the pandemic also have an upstream role in cardiovascular outcomes even among those without COVID-19.

\section{Direct viral infection}

The receptor for SARS-CoV-2 is angiotensin-converting enzyme 2 (ACE2), which is essential to the function of the reninangiotensin system. Following binding of the SARS-CoV-2 spike protein via ACE2, cellular entry is facilitated by the transmembrane protease serine 2 (TMPRSS2) membrane protease and/or endosomal cysteine protease cathepsin L [11]. Virus receptor ACE2 and facilitating proteases are co-expressed on cells throughout the body with a pattern of expression consistent with the clinical symptoms and laboratory findings experienced by patients with COVID-19 [12]. In addition to expression in the respiratory tract and airway, accounting for the characteristic respiratory symptoms of COVID-19, ACE2 is also expressed in the heart and vasculature and direct infection of both has been described.

Direct infection of cardiac tissue with SARS-CoV-2 has been observed and distribution across cell types may be dependent upon expression of ACE2. Upon examination of cardiac tissue from 39 consecutive autopsy cases for patients who died of COVID-19, viral genome was detected in the cardiac tissue of $24(61.5 \%)$ patients and the expression of six pro-inflammatory genes (tumor necrosis growth factor $\alpha$, interferon $\gamma$, chemokine ligand 5, and interleukin (IL) $-6,-8$ and -18) was increased in the cardiac tissue of patients with SARS-CoV-2 compared to those with no evidence of cardiac infection [13]. Consistent with these findings based on the presence of viral genome, one autopsy report described electron microscopic evidence of virus particles in several cell types in the cardiac tissue, including cardiomyocytes, capillary endothelial cells, endocardium endothelial cells, macrophages, neutrophils and fibroblasts [14]. Still other reports did not identify virus particles in cardiac tissue via biopsy or autopsy, but rather in interstitial spaces or the associated endothelial compartment $[15$, 16]. In a study delineating ACE2 expression among different cell types in the heart, Chen et al demonstrated particularly high levels of ACE2 expression on pericytes and close interaction of pericytes and endothelial cells, suggesting that SARSCoV-2 infection of pericytes in the heart may cause rapid dysfunction of nearby endothelial cells [17]. Notably, a recent report describes increased cardiomyocyte-specific ACE2 transcription in diseased cardiac tissue from patients with dilated cardiomyopathy or hypertrophic cardiomyopathy compared to normal human myocardial samples, providing a potential explanation for increased SARS-CoV-2 morbidity and mortality among those with pre-existing cardiovascular disease [18].

Observation of viral particles in the endothelial compartment and endothelial cells of the heart is consistent with other reports in the literature of SARS-CoV-2 infection of the endothelium in other organs. Varga et al found evidence of direct viral infection of endothelial cells accompanied by diffuse endothelial inflammation upon autopsy and microscopic examination of tissue in three patients deceased as a result of COVID-19, concluding that SARS-CoV-2 infection results in endotheliitis in multiple organs through a combination of direct infection and triggering of the host inflammatory response [19]. Similarly, Menter et al presented electron microscopic images of virus particles in an activated glomerular endothelial cell [20]. The presence of susceptible endothelium across multiple organ systems therefore sets the stage for widespread endothelial dysfunction, which has indeed been observed in the lungs, kidneys and hearts of patients with COVID-19 [15, 19, 20].

The fact that SARS-CoV-2 uses ACE2 as its receptor, an important component of the renin-angiotensin system, also has cardiovascular consequences. The renin-angiotensin system has two axes that serve to balance one another and maintain circulatory homeostasis. ACE2 dysfunction disrupts this balance, resulting in increased platelet adhesion and aggregation, 
which in turn increases the risk of thromboembolism [21]. Dysfunction in the renin-angiotensin system is also tightly tied to immunopathology, as described further below.

\section{Immunopathology}

Direct viral infection of the heart and vasculature can result in localized production of pro-inflammatory factors as well as apoptosis and pyroptosis, which serve to increase local inflammatory processes. In concert with evidence of direct viral infection of endothelial cells, Varga et al also found evidence of diffuse endothelial inflammation associated with mononuclear and neutrophilic infiltrates and apoptosis [19]. Evidence suggests that these systemic inflammatory processes, as opposed to those induced locally via direct infection of the myocardium or endothelium, may play a larger role in determining cardiovascular outcomes. For example, in the autopsy study conducted by Lindner et al describing the presence of viral genome in the heart, inflammatory cytokine expression increased with increased viral load, but neither viral load nor virus presence correlated with the presence of inflammatory cells in the myocardium [13]. Inflammatory pathways have a well-established role in acute injury to the myocardium and the response to other respiratory viruses (e.g., influenza) has been shown to increase risk for and/or contribute to the development of heart failure [22].

Early reports regarding the clinical course of COVID-19 out of Wuhan, China, the epicenter of the pandemic, described severe systemic inflammation as a clinical predictor of mortality prompting recognition of cytokine storm as an important consideration among patients with COVID-19 [23, 24]. Cytokine storm is characterized by an overactive immune response and the production and release of excess cytokines into the bloodstream. While pro-inflammatory cytokines are generally beneficial to the management of infection, over-production combined with immediate release into systemic circulation is harmful to the body. Cytokine storm may occur in response to infection, autoimmune conditions, or other diseases and includes symptoms of increased temperature, muscle soreness, joint pain, and if it continues unchecked, can result in multiorgan failure. Mahmudpour et al offer a detailed description of the purported mechanisms underlying hyper-inflammation in the context of COVID-19 [25]. In addition, several publications describe the impact of inflammatory cytokines on the pulmonary system and the role of cytokine storm as a factor leading to clinical deterioration, organ failure and death in COVID-19 [26]. Here we will focus on the consequences of SARS-CoV2-induced inflammation specific to the cardiovascular system.

Whether or not the initial inflammatory response originates in the cardiovasculature, exaggerated systemic inflammation can exacerbate or even cause cardiovascular injury. Increased cytokine levels are associated with myocardial and endothelial damage, abnormal coagulation and thrombus formation [27], much of which is mediated via the impact of inflammatory molecules on endothelial cells. The extent to which the endothelium mediates the multi-organ effects of SARS-CoV-2 prompted a recent publication in the European Heart Journal by Peter Libby and Thomas Luscher that proposes re-conceptualizing COVID-19 as an endothelial disease, noting the role of the endothelium in even the disease's characteristic damage to the lungs [28]. In patients with COVID-19, heightened levels of pro-inflammatory cytokines IL-2, $-6,-7$ and -8 are consistently observed and their production correlates with disease severity [21]. Circulation of these pro-inflammatory cytokines activates the endothelium, creating an environment within blood vessels that is pro-coagulant/pro-aggregatory, anti-fibrinolytic, pro-inflammatory, pro-oxidant, and characterized by impaired barrier function and vasoconstriction [28]. Acute release of pro-inflammatory cytokines, including IL-1, IL-6 and plasminogen activator inhibitor (PAI-1), have also been associated with increased risk of heart failure with preserved ejection fraction [21], and chronic inflammation is known to be an important pathogenic driver of heart failure in general [29].

\section{Hypoxemia}

The endothelial dysfunction described above may be further exacerbated by hypoxemia, the likelihood of which increases with progressive decline in lung function associated with COVID-19. The hypoxemic effects of reduced oxygen intake are further compounded by the increased demands for oxygen necessitated by the host response to infection, potentially resulting in cardiac injury [30]. Hypoxemia is also linked to endothelial damage and has been shown to increase risk for thrombosis in patients with COVID-19. As endothelial cells are responsible for maintaining the vasculature, including vessel wall permeability, dysfunction of endothelial cells in the pulmonary vasculature gives rise to thrombotic processes that may increase the risk of acute respiratory distress syndrome (ARDS). Hypoxia-mediated hypercoagulability was identified as an important factor in ARDS in the context of COVID-19 compared to other causes of ARDS [31]. Further, lung lymph vessels are especially susceptible to oxidative stress and apoptosis of lung microvascular cells has been observed [32].

\section{Medication-induced cardiac toxicity}

Medication-induced toxicity represents another potential contributor to COVID-19 cardiovascular damage. At the start of the COVID-19 pandemic, medical providers found themselves facing an unfamiliar disease without evidence-based treatment strategies and have instead had to focus on interventions designed to ameliorate symptoms and/or off-label use of drugs previously approved for other purposes. Some such drugs must be used with particular caution due to the potential for cardiac toxicity, especially in patients with pre-existing cardiovascular disease or other comorbidities. For example, hydroxychloroquine use with or without azithromycin was quickly adopted by many medical facilities based on early evidence of efficacy for reducing viral load. However, the cardiac toxicity of hydroxychloroquine and chloroquine, a related anti-malarial medication, has been known for some time. Use of chloroquine and hydroxychloroquine has been associated with atrial and ventricular arrhythmias, fascicular block and syncope, and QT interval prolongation, which can increase the risk of in- 
duced polymorphic ventricular tachycardia and sudden cardiac death [33]. Examination of cardiac function in patients who received hydroxychloroquine for COVID-19 has had mixed results. In one recent trial, patients who received hydroxychloroquine and azithromycin had severe QTc prolongation [34], and in another, those who received both hydroxychloroquine and azithromycin were significantly more likely to suffer cardiac arrest than those who did not receive either drug [35]. Fewer such events were reported in a publication in which patients were systematically excluded from receiving hydroxychloroquine and azithromycin for cardiac contraindications or the potential risk for drug interactions [36], which may represent the development of improved understanding of risk vs. benefit. In a recent review of major developments related to the use of hydroxychloroquine for COVID-19 treatment and prophylaxis, Singh et al conclude that until in progress, well-designed, randomized controlled trials identify specific subgroups likely to benefit from hydroxychloroquine it should be used for patients with COVID-19 only under investigational trial settings with close safety monitoring [37].

Numerous drug trials are currently underway to investigate the utility of anti-inflammatory, anti-rejection, anti-complement and anti-neoplastic agents for the treatment of COVID-19 [38]. In a systematic review of the therapies currently under investigation, Campbell et al note the importance of considering the potential cardiotoxicity of these agents given that adverse effects on the cardiovascular system have significant potential to increase risk of morbidity and mortality [38]. Current evidence suggests that anti-inflammatory agents that directly inhibit the IL-1 and JAK pathways as well as complement inhibitors are the least likely of all agents under consideration to result in cardiotoxicity, although there are relatively little data available on the cardiovascular profile of the complement inhibitors under investigation [38].

It is also important to understand and recognize the sideeffects of certain drugs that specifically target the cardiovascular system. Such drugs include ACE inhibitors and angiotensin receptor blockers (ARBs). Siddamreddy et al observed upregulation of ACE2 in patients taking either class of medications, specifically in cardiomyocytes [39]. Some have theorized that upregulation of ACE2 could lead to patient deterioration and progression of the disease, but there are little data to support this theory. At present, the American College of Cardiology and the American Heart Association recommend continued use of ACE inhibitors and ARBs for patients that are already on those medications [39]. Driggin et al provide an excellent summary of antiviral and other therapies currently under investigation for use in COVID-19 and their potential interactions with common cardiovascular medication classes as well as known cardiovascular adverse effects [40], which is likely to prove useful to clinicians evaluating the utility of such therapies in the absence of clear clinical guidance.

\section{Indirect cardiovascular consequences of the COVID-19 pandemic}

It is worth noting the likelihood that the ongoing COVID-19 pandemic has cardiovascular consequences even for those who are not infected with SARS-CoV-2. The European Society for Cardiology has dubbed these cardiovascular effects "collateral damage of COVID-19" and points to patient vulnerability, lack of assurance of safe hospital environments, increased strain on emergency services and reprioritization of hospital resources as its causes [41]. For example, in a comparison of hospital admissions for heart failure in Germany during the pandemic to control time periods earlier in 2020 and the same time frame in 2019, Bollmann et al observed fewer admissions for heart failure, shortened hospital stays and increased in-hospital mortality among those admitted for heart failure and no diagnosis of COVID-19 from March 13 to April 30, 2020 compared to the control periods [42]. This is consistent with reports from both Italy and the USA describing a significant decrease in hospitalizations for acute myocardial infarction in the pandemic era $[43,44]$. Gori et al described additional implications for cardiovascular disease among patients without COVID-19, including postponement of elective procedures, delays before and during hospital admission, reduced access to care due to fear of contagion, shortened diagnostic protocols as hospital capacity becomes overwhelmed, and risk of diagnostic errors [45].

\section{Clinical Cardiovascular Presentations}

Clinically, the cardiovascular consequences of COVID-19 may present as myocarditis with or without arrhythmia, endothelial dysfunction and thrombosis, acute coronary syndromes and heart failure. Presentation can vary widely and may or may not be typical of the condition in an individual without COVID-19.

\section{Myocarditis}

Myocarditis, or direct inflammation of the myocardium and myocardial injury without ischemic origin, can result in reduced ability to pump blood, irregular electrical activity and arrhythmia. Numerous studies suggest that the pathophysiology of viral myocarditis in the context of SARS-CoV-2 can be attributed to direct injury of infected cardiomyocytes in combination with systemic inflammation, as described above. The pathology of myocarditis in the context of COVID-19 is debated, but a report by Fox et al sheds light on the subject [46]. Upon autopsy, histological examination of the heart from a possible case of myocarditis was marked by an unusually low concentration of lymphocytes in the tissue. The authors suggest that this finding may support the notion that direct infection of pericytes may trigger endothelial damage, which could in turn lead to cell death in the heart [46]. Consistent with this notion, Lindner et al reported that upon autopsy, neither virus presence nor viral load was correlated with the presence of inflammatory cells in the myocardium [13].

The prevalence of SARS-CoV-2 myocarditis is difficult to estimate due to a number of confounding factors, including a lack of diagnostic tools in initial reports, incomplete laboratory testing protocols and difficulty discerning the symptoms of myocarditis from other COVID-19 symptoms. Early on in 
the pandemic, rationing of tests resulted in preferential testing of samples for other respiratory pathogens before testing for COVID-19. If a patient tested positive for another pathogen that could explain the symptoms, the individual was never tested for COVID-19. However, in one study, the rate of coinfection with SARS-CoV-2 and at least one other respiratory pathogen was $20.7 \%$, suggesting that a lack of thorough testing would likely miss as many as one in five cases of COVID19-related myocarditis [47].

COVID-19 patients with myocarditis may present with a wide range of symptoms and conditions. For example, Kim et al reported a case of a 21-year-old patient with classic COVID-19 symptoms, including difficulty breathing, shortness of breath and fever [48]. Upon further examination, computed tomography (CT) scans of the chest showed ground-glass opacity in both lungs as well as general thickening of the myocardium. Cardiac troponin levels were also suggestive of cardiac injury. Further imaging, including echocardiography and cardiac magnetic resonance imaging (c-MRI), ultimately confirmed the diagnosis of myocarditis. While the patient outwardly expressed mild signs of dyspnea, fatigue and fever, clinical diagnostic workup revealed more severe complications of viral infection [48]. Without the presence of any more specific cardiovascular symptoms, it is likely that many such cases of myocarditis are undiagnosed. Another common clinical presentation indicative of cardiac involvement in COVID-19 includes chest pain and tightness. In the first published report of COVID-19 complicated by fulminant myocarditis, Zeng et al describe a 63-yearold male patient with symptoms including white sputum, high fever and chest tightness [49]. The patient also had elevated cardiac troponin levels and pneumonia. The patient's condition continued to deteriorate and there was evidence of increased levels of pro-inflammatory cytokines. Imaging showed thickening of the left ventricle as well as an abnormally low left ventricle ejection fraction and the patient was diagnosed with myocarditis. Despite signs of improvement with the left ventricle returning to normal size, the patient developed a secondary infection that ultimately led to cardiac dysfunction on the right side of the heart and the patient died after 33 days of hospitalization [49].

Two recent studies suggest that in some cases of myocarditis, individuals may have no symptoms at all. Puntmann et al conducted c-MRI on an unselected sample of 100 individuals who had recovered from COVID-19, the majority of whom recovered at home, and detected evidence of cardiac involvement in $78 \%$ of participants, most often presenting as myocardial inflammation [9]. Importantly, cardiac involvement was independent of severity or extent of COVID-19 disease and relatively few individuals reported any cardiac symptoms, even upon direct questioning. This finding is supported by a report by Rajpal et al demonstrating myocardial involvement in $46 \%$ of 26 competitive college athletes with COVID-19 who had mild or no symptoms, suggesting that frequent cardiac involvement may occur even among those who are otherwise young and healthy [8].

Finally, reports in the literature suggest that viral myocarditis may contribute to COVID-19-related fatalities. In a study of 150 patients by Ruan et al, 68 deaths were recorded, five of which $(7 \%)$ were attributed to myocarditis. This study shows that a minor percentage of COVID-19 patients who died presented with viral myocarditis [24].

\section{Arrhythmia}

Arrhythmia is a classic clinical manifestation of myocarditis and may be particularly prevalent among individuals with COVID-19. In an early description of 138 hospitalized patients in Wuhan, China, Wang et al reported that more than $40 \%$ of transfers to the intensive care unit (ICU) among patients hospitalized for COVID-19 were attributable to arrhythmia [50]. Although this report was unable to ascribe the cause of arrhythmia to myocarditis, a later report by Siripanthong et al indicated that $78.7 \%$ of patients with COVID-19 who had been diagnosed with myocarditis showed signs for some form of arrhythmia [51].

In a review of arrhythmia in COVID-19, Babapoor-Farrokhran et al identified sinus bradycardia as the most commonly reported form of arrhythmia associated with COVID-19, but also described several reports of atrial tachyarrhythmia as well as ventricular arrhythmias and noted that case reports in the published literature are unlikely to represent actual prevalence [52]. In a global survey conducted by the Heart Rhythm Society in the spring of 2020, severe sinus bradycardia and complete heart block were the most commonly reported bradyarrhythmias associated with COVID-19 [53]. Reports suggest that sinus bradycardia can occur even in patients with COVID-19 and normal echocardiography and cardiac biomarkers [54], and can persist for as long as 2 weeks [55]. Several reports of atrial tachyarrhythmia in the context of COVID-19, including atrial fibrillation, atrial flutter and atrial tachycardia, have also been published [52]. In the global Heart Rhythm Society survey, atrial fibrillation was the most common arrhythmia reported overall [53].

Evidence suggests that several life-threatening ventricular arrhythmias can also occur in COVID-19 [52]. Respondents to the global Hearth Rhythm Society survey reported sustained monomorphic ventricular tachycardia, polymorphic ventricular tachycardia/Torsade de Pointes, ventricular tachycardia/ ventricular fibrillation arrest and pulseless electrical activity [53]. There is some evidence to suggest that ventricular arrhythmias are more often associated with elevated cardiac biomarkers and/or use of medications that can prolong the QT interval [34, 56-59]. Several Heart Rhythm Society survey respondents reported discontinuation of hydroxychloroquine/ chloroquine and azithromycin as a result of prolonged QT interval or Torsade de Pointes [53].

\section{Endothelial dysfunction and thrombosis}

The endothelium comprises the cells lining the vasculature and functions to control the flow of fluids and substances into and out of tissues. In general, endothelial dysfunction is marked by the decreased production of nitric oxide as well as disturbed regulation of the relaxation and contraction of the vasculature [60]. Endothelial dysfunction is particularly important in 
COVID-19, likely contributing to disease manifestations in several organ systems [28]. It is purported that endothelial dysfunction in COVID-19 may be caused by viral entry through ACE2, thereby causing an abnormal response of the renin-angiotensin system and activating endothelial cells. The resulting pro-inflammatory and pro-coagulatory milieu observed in patients with COVID-19 is characterized by increased levels of inflammatory cytokines and chemokines, von Willibrand factor antigen and activity, factor VIII, and acute phase reactants (e.g., C-reactive protein, D-dimer) [61].

COVID-19-related endothelial dysfunction manifests via a pro-coagulatory state and thrombotic activity. In a report detailing the cases of 12 patients in Hamburg, Germany, death was the result of pulmonary embolism in four cases and deep vein thrombosis was observed in seven [62]. Additional studies have shown that such thrombotic complications are remarkably common, with one such study by Klok et al finding that nearly one-third of patients with COVID-19 admitted to the ICU at three Dutch hospitals had at least one thrombotic event [63]. Bilaloglu et al reported a slightly lower frequency in their case series of 3,334 consecutively hospitalized patients with COVID-19, not limited to those requiring care in the ICU, in New York City where 16\% of individuals had at least one thrombotic event [64].

It also appears that COVID-19-related mortality can be linked to endothelial dysfunction and coagulopathy. Wichmann et al reviewed data from 191 patients with COVID-19 and found that $50 \%$ of patients who succumbed had evidence of coagulopathy and that patients with D-dimer levels greater than $1,000 \mu \mathrm{g} / \mathrm{L}$ were more likely to have fatal outcomes [62]. In addition to the endothelial activation described above, Wichmann et al also describe hypoxemia, a likely occurrence in individuals experiencing severe respiratory symptoms, as a possible trigger for thrombus formation as hypoxic conditions in animal models showed facilitated thrombus formation [62]. Thrombus formation can also be exacerbated by systemic inflammation.

In addition to the more generalized endothelial dysfunction described above, endotheliitis is also of particular concern in the context of COVID-19. Data published by Mosleh et al indicate the direct infection and presence of viral particles in endothelial cells causing inflammation of the endothelium [65]. This condition is particularly relevant to the development of pulmonary edema, as shown by Teuwen et al [66]. Data from Huertas et al further corroborate this theory with histological evidence of viral particles and inflammatory cell infiltrates in the endothelium [67]. Dramatic changes in the structure of the endothelium as a result of this inflammatory state result in loosening of the endothelial gaps and consequent vascular leakage and build-up of fluid in the alveolar space.

While patients of all demographics can be affected by virus-associated endothelial dysfunction, males and the elderly appear to be disproportionately affected. Under even normal circumstances, vasoconstriction is heightened and vasodilation is decreased due to endothelial dysfunction in the elderly. Other risk factors for endothelial dysfunction include pre-existing cardiovascular conditions, hypertension and diabetes [68]. In addition, Zhang et al propose that the emerging association between vitamin D deficiency and poor outcomes for COVID-19 patients may be mediated, at least in part, via the role of the vitamin D receptor in normal endothelial function [69].

\section{Acute coronary syndromes}

Like several other viral respiratory infections, COVID-19 appears to increase risk for acute coronary syndromes, including myocardial infarction with or without ST-segment elevation [70$72]$. Interestingly, in a report of 28 cases published by Stefanini et al, ST-segment elevation myocardial infarction represented the first clinical manifestation of COVID-19 for 24 (86\%) of patients, while it developed only after hospitalization for other COVID-19 symptoms in the remaining four cases [72].

That COVID-19 would increase risk for acute coronary syndrome is unsurprising given that a known risk factor for cardiac injury is lack of oxygen, as occurs with severe respiratory symptoms, and increased oxygen demand, as occurs during the host response to infection. It is difficult to assess the true prevalence of myocardial infarction, however, as its identification is challenged by symptoms of dyspnea and other comorbidities. In some cases, this dyspnea itself may place significant strain on the myocardium. In addition, cardiac troponin levels increase with both acute myocardial infarction and myocarditis, making it difficult to determine the actual cause. Knight et al used c-MRI to determine mechanism of injury among 29 individuals who had been hospitalized for COVID-19 and had elevated troponin levels indicative of unexplained myocardial injury with no cause for previous myocardial scarring [73]. Among 20 patients with an identifiable mechanism, ischemic heart disease-related pathology was half as common as non-ischemic, myocarditis-like pathology [73].

The characteristic over-reactive immune response and prothrombotic state undoubtedly contributes to the occurrence of acute coronary syndrome in patients with COVID-19. For example, among 533 patients with COVID-19 that had a thrombotic event while hospitalized in New York City, more than half experienced myocardial infarction [64]. More specifically, ST-elevated myocardial infarction in patients with COVID-19 may be attributable to microthrombi formation, as shown by Guagliumi et al [74]. In one patient with ST-elevated myocardial infarction who ultimately succumbed to COVID-19, multiorgan failure was associated with prothrombin activation fragments and examination post-mortem showed microthrombi in the kidney, spleen and walls of the ventricles [74].

Cardiac arrest and sudden cardiac death also appear in connection with COVID-19, although prevalence is much harder to characterize for a number of reasons, including the indirect consequences of the pandemic on emergency infrastructure and care-seeking behavior $[33,70]$. In one report of three patients with COVID-19 who suffered cardiac arrest by Shirazi et al, perhaps the most notable similarity was that cardiac arrest occurred after improvement in disease course among individuals not thought to be at risk for developing cardiac arrest [75]. All three patients had been treated with a combination of antiviral medications and hydroxychloroquine, prompting the investigators to note the importance of closely monitoring cardiovascular function in all patents with COVID-19 and particularly those receiving hydroxychloroquine [75]. Mazzanti et 
al observed changes in QT interval even when hydroxychloroquine was administered to patients with COVID-19 only for a short duration ( $2 \mathrm{~g}$ over 5 days) [76].

\section{Heart failure}

Heart failure is a relatively common cardiovascular complication of COVID-19 [77]. In two initial case series out of China, heart failure was reported in $23-24 \%$ of patients and was significantly more common among those who died than those who recovered $[78,79]$. In patients with COVID-19, heart failure is thought to occur either as the result of direct viral infection, inflammation and cardiac fibrosis or by unmasking or exacerbating pre-existing, sub-clinical disease [22]. Reports suggest that although right ventricular abnormalities and left ventricular diastolic dysfunction are relatively common among individuals with COVID-19, left ventricular ejection fraction tends to be normal suggesting that heart failure with preserved ejection fraction (HFpEF) is of particular importance in the context of COVID-19 [5, 22, 80]. Heart failure in the context of COVID-19 can also occur secondary to myocarditis, ischemia, and/or arrhythmia [81].

\section{Cardiovascular Prognostic Indicators}

That COVID-19 morbidity and mortality is higher among those with cardiovascular involvement compared to those without has been known since relatively early in the course of the pandemic [71]. In a number of clinical case series from hospital settings, cardiovascular complications (e.g., arrhythmia) were significantly more common and biomarkers of myocardial injury (e.g., cardiac troponin) were significantly higher in patients requiring ICU admission and/or ventilation than in those who did not [33]. Useful biomarkers of myocardial injury and cardiovascular prognosis include hs-troponin and natriuretic peptide (NP). Upon retrospective analysis of hs-troponin levels in 523 patients admitted to the hospital with COVID-19 in Italy, Michela et al identified hs-troponin as an independent risk factor for mortality with a hazard ratio (HR) of 1.004 for each 10-point increase in hs-troponin level ( $\mathrm{P}<$ 0.0001 ) [82]. Similarly, Shah et al examined hs-troponin levels in 309 patients admitted to a US hospital with COVID-19 and found a direct correlation between cardiac troponin I (cTnI) levels and need for intubation, dialysis, transfer to the ICU and death [7]. Arcari et al suggested that both hs-troponin and NP may be particularly useful biomarkers for clinical pneumonia risk stratification [6]. In their study of 111 patients with COVID-19 pneumonia admitted to the hospital, elevated hstroponin and/or NP was associated with several markers of poor clinical function, including reduced $\mathrm{PaO}_{2} / \mathrm{FIO}_{2}$ and right ventricular function, and a significantly increased risk of inhospital mortality [6].

Markers of endotheliopathy can also serve as useful prognostic indicators. In a study by Goshua et al, which assessed endothelial cell and platelet activation among 68 patients, P-selectin and thrombomodulin were found to be viable bio- markers for endothelial cell activation and $\mathrm{SCD} 40 \mathrm{~L}$ was identified as viable biomarker for platelet activation [83]. Fatalities related to endothelial dysfunction were noted and a marked increase of P-selectin and sCD40L was observed among patients who required intensive care. The study also found that patients with low thrombomodulin levels tended to have better outcomes than those with elevated levels. D-dimer as a marker for thrombosis also has prognostic value. In a study of 3,334 consecutively hospitalized patients with COVID-19 in New York City, D-dimer level at presentation was independently associated with risk of thrombotic events [64].

\section{Clinical Recommendations for Cardiovascular Screening and Treatment}

Special considerations are recommended for patients that present with cardiovascular symptoms along with classic symptoms of COVID-19 (i.e., respiratory symptoms, fever, dyspnea). Obtaining a thorough patient history is important for detecting symptoms and implementing management strategies early in the course of disease. While not all cases have severe outcomes, evidence suggest that it may still be beneficial to test for biomarkers indicative of cardiac injury, such as cardiac troponin and NP [51]. In addition, imaging and other interventional cardiology approaches should be considered when there are concerns for myocarditis or acute coronary syndrome.

In a report of echocardiographic findings from 66 highrisk cases, Schott et al described frequent right ventricle dilation and increased wall thickness [84]. The American College of Cardiology and American Society of Echocardiography recommend that echocardiographic examinations in the context of COVID-19 are performed in a manner that reduces sonographer exposure time and are conducted only if it is likely that results will change clinical management [85]. Cau et al compared the use of echocardiography and c-MRI for use in the context of COVID-19 ultimately concluding a complimentary role for the two modalities whereby the use of echocardiography for a rapid bedside diagnosis can be further refined via c-MRI [86]. Kociol et al also found c-MRI to be useful for detecting myocarditis [87]. Classic imaging for myocarditis includes excess wall thickness and widening of the chambers. While the feasibility of c-MRI can be limited by local availability, cost and time constraints, and other intrinsic and extrinsic patient-specific factors, its utility in determining the presence and extent of myocardial tissue damage makes it a valuable diagnostic and prognostic tool in the context of COVID-19. Kochi et al also recommend use of c-MRI to assess the extent of myocardial scarring as a means to determine arrhythmia risk among patients who recover from COVID-19, but have evidence of myocardial injury [33].

Further diagnostic tests that can be administered if there are concerns for myocardial infarction include an electrocardiogram to measure electrical activity, angiogram with coronary catheterization to identify areas of arterial blockage and laboratory blood testing to assess biomarkers indicative of cardiac damage (e.g., troponin). The actual treatment of myocardial infarction is debated in the context of COVID-19. The 
American College of Cardiology states that fibrinolysis may be used for patients with a low-risk ST-elevated myocardial infarction. However, percutaneous coronary intervention remains the standard of care in patients with COVID-19 [88]. Although a recent multi-national case series regarding clinical outcomes among patients with COVID-19 presenting with ST-segment elevation myocardial infarction found a high rate of stent thrombosis among patients who were treated with percutaneous coronary intervention, which suggests the potential need to adapt management for this condition in patients with COVID-19 [89].

At present, remdesivir represents the only anti-viral medication with evidence of efficacy in COVID-19. Although not specific to cardiovascular function, results of the Adaptive COVID-19 Treatment Trial (ACTT-1) published in May 2020 demonstrated that remdesivir shortened time to recovery in adults hospitalized with COVID-19 who had evidence of lower respiratory tract infection compared to placebo control in a double-blind, randomized, placebo-controlled trial [90]. More recently, the FDA granted an emergency use authorization to Regeneron Pharmaceuticals Inc. for an antibody cocktail including the monoclonal antibodies casirivimab and imdevimab targeting the SARS-CoV-2 spike protein based on evidence that intravenous administration of the cocktail reduces COVID-19-related hospitalization or emergency room visits among those at high risk for disease progression compared to placebo [91]. The use of medication to manage endothelial dysfunction, coagulopathy and the hyper-inflammatory response associated with COVID-19 may have further beneficial effects for the cardiovascular system as long as those same medications are not themselves associated with cardiotoxicity. For example, some have postulated that the combined anti-viral and anti-inflammatory properties of the FDA-approved drug ivermectin account for its promise as a COVID-19 treatment, which is currently the subject of a number of clinical investigations $[92,93]$. Numerous other anti-inflammatory therapies are currently being investigated for mitigation of the effects of systemic inflammation and cytokine storm in patients with COVID-19. Immunomodulatory agents that directly target the specific cytokines involved in the host response to SARSCoV-2 are of particular interest. In serious cases, these immunomodulatory agents can alleviate hyperinflammation. For example, the antibody therapy tocilizumab and ulinastatin are drugs that specifically block IL-6 and may be useful for patients with COVID-19 suffering from over-inflammatory processes [38]. Other antiviral drugs tested in clinical trials for the treatment of COVID-19 have to date proven ineffective and there is some speculation that a combination of immunomodulatory agents and antiviral therapies could yield the best treatment for inflammation in COVID-19 patients [94]. In addition, administration of the steroid dexamethasone was shown in a randomized controlled trial to reduce mortality among those with severe respiratory disease requiring mechanical ventilation or oxygen administration [95]. Until effective treatment strategies are well-defined, treatment providers are urged to maintain awareness of the potential for cardiotoxicity that may not yet be fully appreciated, but could be particularly harmful in the context of COVID-19 [38]. Driggin et al provide an excellent summary of putative therapies for COVID-19 along with known interactions with existing cardiovascular medications, recommendations for dose adjustments and tips for effective monitoring [40].

Of note, aspirin represents an existing cardiovascular medication with promise for addressing some of the cardiovascular symptoms of COVID-19. In a retrospective study of 412 patients hospitalized in the US for COVID-19, receipt of aspirin within $24 \mathrm{~h}$ of admission or 7 days prior to admission was associated with a significantly decreased risk of mechanical ventilation, ICU admission and in-hospital mortality [96].

It is worth noting that in addition to those with common risk factor for cardiovascular disease or known cardiovascular complications of SARS-CoV-2 infection, it may also be necessary to screen for cardiovascular dysfunction among individuals with relatively mild or even asymptomatic infection if they plan to engage in high-intensity physical activity (e.g., competitive athletes). Rajpal et al conducted c-MRI on 26 collegiate-level competitive athletes who had COVID-19 associated with either mild or no symptoms and found that $12(46 \%)$ had evidence of ongoing myocarditis and/or prior myocardial injury suggesting that this screening modality may be useful for guiding decisions about returning to competitive sports participation [8]. Evidence suggests that cardiac involvement is common even among those with mild or no symptoms of COVID-19 [9], and healthcare providers who cared for patients with COVID-19 during the peak of the pandemic in Italy suspect that sudden cardiac death may have been the cause of death for patients with only mild symptoms who were not hospitalized, but found dead at home during quarantine [33]. As a result, Kochi et al recommend evaluation of myocardial biomarkers in all patients with COVID-19 to accommodate risk stratification and early intervention [33]. Whether such a broad screening strategy is warranted or even feasible remains to be determined.

The American Heart Association COVID-19 CVD registry launched in April is positioned to address many of the uncertainties described above. Intended to function as a hospital quality improvement program, it will also result in an aggregated national dataset to inform best practices for the evaluation and management of COVID-19 with special consideration of the cardiovascular impacts of the disease. With approximately 150 participating hospitals across the US, data are rapidly accumulating and bringing greater certainty regarding the clinical management of COVID-19 ever nearer [10].

\section{Long-Term Cardiovascular Consequences}

Considerable evidence points to the possibility of long-term cardiovascular consequences for those who recover from COVID-19. There is evidence of long-term cardiovascular dysfunction among individuals who recovered from infection with SARS-CoV. In a study of 25 patients who had recovered from SARS-CoV 12 years earlier, 44\% had evidence of cardiovascular abnormalities and lipid metabolism dysregulation [97]. Although the mechanism accounting for this finding is not understood, similarity between SARS-CoV and the novel SARS-CoV-2 suggests the potential for long-term cardiovas- 
cular consequences among those who recover. For example, the possibility exists that myocardial injury as a result of COVID-19 will result in atrial or ventricular fibrosis and a subsequent increased long-term risk for cardiac arrhythmias [33]. A number of investigators similarly suggest the potential for COVID-19 survival to ultimately be identified as an independent risk factor for later development of heart failure and other long-term cardiovascular sequelae including cardiac hypertrophy, cardiac fibrosis, chronic right heart failure, decreased cardiac output, diastolic dysfunction and pulmonary hypertension, particularly among those recovering from severe illness characterized by profound hypoxemia, respiratory failure and thromboembolic complications $[22,29]$. To mitigate these risks, Kochi et al recommend the use of c-MRI to stratify patents for follow-up [33]. The value of such an approach is supported by a report by Puntmann et al in which c-MRI was conducted on an unselected cohort of 100 German patients who had recovered from COVID-19 [9]. Among this cohort, there was evidence of cardiac involvement in $78 \%$ of individuals and ongoing myocardial inflammation in $60 \%$ of individuals, both of which were independent of any pre-existing conditions, severity of the acute COVID-19 illness and time from original diagnosis [9]. Similarly, Knight et al used c-MRI to assess myocardial damage among 29 individuals in early convalescence who were troponin-positive during hospitalization, but had unexplained myocardial injury and no cause for previous myocardial scarring [73]. Mechanisms of myocardial injury were identified for $20(69 \%)$ patients via c-MRI and included non-ischemic heart disease-related (38\%), ischemic heart disease-related (17\%), and dual ischemic and non-ischemic (14\%) pathology. Non-ischemic injuries frequently appeared to be myocarditis-like and lack of associated edema suggests that the myocarditis-like scarring observed may be permanent, leading the authors to conclude the myocardial injury is common with potentially long-lasting effects, even among those who did not experience acute coronary syndrome or pulmonary emboli [73].

\section{Conclusion}

Characterizing COVID-19 as an endothelial disease, as suggested by Libby and Luscher [28], more appropriately accounts for the systemic effects of SARS-CoV-2, which impacts the cardiovascular system in much the same way it impacts the respiratory system, but is less well known for doing so. As such, medical personnel must be vigilant in their attention to possible cardiovascular symptoms, take appropriate steps for clinical diagnosis and be prepared for long-term ramifications of myocardial injury sustained as a result of COVID-19. The understanding of the relationship between cardiovascular disease and COVID-19 is rapidly evolving. We have tried to cover key aspects of this relationship as understood in the fall of 2020, but fully recognize that newer findings will be published by the time this paper appears in print and certain aspects of this review may become rapidly outdated. For those readers interested in the topic, please keep a close eye on new papers as they appear in the medical literature.
Ultimately, preventing the direct and indirect, short- and long-term cardiovascular consequences of COVID-19 will depend on controlling the pandemic and preventing additional infections from occurring. The Pfizer-BioNTech COVID-19 Vaccine, which has a reported $95 \%$ efficacy 28 days after the first dose, has received emergency use authorization from authorities in the US and UK [98, 99]. Approval of a Moderna vaccine with $94 \%$ reported efficacy is also anticipated [100]. Combined with ongoing efforts to limit person-to-person spread, launch of vaccination campaigns appears to have made such control a real possibility.

\section{Acknowledgments}

None to declare.

\section{Financial Disclosure}

The study was funded in part by the Marylou Ingram Endowment at Huntington Medical Research Institutes, the Francis Bacon Fund and the John and Lucille Crum Medical Research Endowment to Huntington Medical Research Institutes.

\section{Conflict of Interest}

The authors declare that they do not have a conflict of interest.

\section{Author Contributions}

Sidhant Umbrajkar researched and collected the reference papers, wrote the initial draft of the manuscript and edited the manuscript. Dr. Robert A. Kloner conceived of the manuscript, helped with the initial outline, reviewed references with Sidhant Umbrajkar, re-wrote and edited portions of the manuscript. Rachel V. Stankowski researched, edited and re-wrote sections of the paper. Dr. Shereif Rezkalla helped conceive of the manuscript and edited the manuscript.

\section{Data Availability}

The authors declare that data supporting the findings of this study are available within the article.

\section{References}

1. Division of Viral Diseases, National Center for Immunization and Respiratory Diseases (NCIRD), Centers for Disease Control and Prevention (CDC), US Department of Health and Human Services. Scientific Brief: SARSCoV-2 and Potential Airborne Transmission. https:// www.cdc.gov/coronavirus/2019-ncov/more/scientificbrief-sars-cov-2.html. Updated 5 October 2020. Accessed 
11 October 2020.

2. Li B, Yang J, Zhao F, Zhi L, Wang X, Liu L, Bi Z, et al. Prevalence and impact of cardiovascular metabolic diseases on COVID-19 in China. Clin Res Cardiol. 2020;109(5):531-538.

3. Barber C. COVID-19 can wreck your heart, even if you haven't had any symptoms. Scientific American. Aug 31, 2020.

4. Zheng YY, Ma YT, Zhang JY, Xie X. COVID-19 and the cardiovascular system. Nat Rev Cardiol. 2020;17(5):259260.

5. Szekely Y, Lichter Y, Taieb P, Banai A, Hochstadt A, Merdler I, Gal Oz A, et al. Spectrum of cardiac manifestations in COVID-19: a systematic echocardiographic study. Circulation. 2020;142(4):342-353.

6. Arcari L, Luciani M, Cacciotti L, Musumeci MB, Spuntarelli V, Pistella E, et al. Incidence and determinants of high-sensitivity troponin and natriuretic peptides elevation at admission in hospitalized COVID-19 pneumonia patients. Internal and Emergency Medicine. 2020;28:110.

7. Shah P, Doshi R, Chenna A, Owens R, Cobb A, Ivey H, Newton S, et al. Prognostic value of elevated cardiac troponin I in hospitalized COVID-19 patients. Am J Cardiol. 2020;135:150-153.

8. Rajpal S, Tong MS, Borchers J, Zareba KM, Obarski TP, Simonetti OP, Daniels CJ. Cardiovascular magnetic resonance findings in competitive athletes recovering from COVID-19 infection. JAMA Cardiol. 2021;6(1):116-118.

9. Puntmann VO, Carerj ML, Wieters I, Fahim M, Arendt C, Hoffmann J, Shchendrygina A, et al. Outcomes of cardiovascular magnetic resonance imaging in patients recently recovered from coronavirus disease 2019 (COVID-19). JAMA Cardiol. 2020;5(11):1265-1273.

10. Alger HM, Rutan C, Williams JHt, Walchok JG, Bolles M, Hall JL, Bradley SM, et al. American Heart Association COVID-19 CVD registry powered by get with the guidelines. Circ Cardiovasc Qual Outcomes. 2020;13(8):e006967.

11. Hoffmann M, Kleine-Weber H, Schroeder S, Kruger N, Herrler T, Erichsen S, Schiergens TS, et al. SARSCoV-2 cell entry depends on ACE2 and TMPRSS2 and is blocked by a clinically proven protease inhibitor. Cell. 2020;181(2):271-280 e278.

12. Liu PP, Blet A, Smyth D, Li H. The science underlying COVID-19: implications for the cardiovascular system. Circulation. 2020;142(1):68-78.

13. Lindner D, Fitzek A, Brauninger H, Aleshcheva G, Edler C, Meissner K, Scherschel K, et al. Association of cardiac infection with SARS-CoV-2 in confirmed COVID-19 autopsy cases. JAMA Cardiol. 2020;5(11):1281-1285.

14. Dolhnikoff M, Ferreira Ferranti J, de Almeida Monteiro RA, Duarte-Neto AN, Soares Gomes-Gouvea M, Viu Degaspare N, Figueiredo Delgado A, et al. SARS-CoV-2 in cardiac tissue of a child with COVID-19-related multisystem inflammatory syndrome. Lancet Child Adolesc Health. 2020;4(10):790-794.

15. Fox SE, Li G, Akmatbekov A, Harbert JL, Lameira FS, Brown JQ, Vander Heide RS. Unexpected features of cardiac pathology in COVID-19 infection. Circulation. 2020;142(11):1123-1125.

16. Tavazzi G, Pellegrini C, Maurelli M, Belliato M, Sciutti F, Bottazzi A, Sepe PA, et al. Myocardial localization of coronavirus in COVID-19 cardiogenic shock. Eur J Heart Fail. 2020;22(5):911-915.

17. Chen L, Li X, Chen M, Feng Y, Xiong C. The ACE2 expression in human heart indicates new potential mechanism of heart injury among patients infected with SARSCoV-2. Cardiovasc Res. 2020;116(6):1097-1100.

18. Tucker NR, Chaffin M, Bedi KC, Jr., Papangeli I, Akkad $\mathrm{AD}$, Arduini A, Hayat S, et al. Myocyte-specific upregulation of ACE2 in cardiovascular disease: implications for SARS-CoV-2-mediated myocarditis. Circulation. 2020;142(7):708-710.

19. Varga Z, Flammer AJ, Steiger P, Haberecker M, Andermatt R, Zinkernagel AS, Mehra MR, et al. Endothelial cell infection and endotheliitis in COVID-19. Lancet. 2020;395(10234):1417-1418.

20. Menter T, Haslbauer JD, Nienhold R, Savic S, Hopfer H, Deigendesch N, Frank S, et al. Postmortem examination of COVID-19 patients reveals diffuse alveolar damage with severe capillary congestion and variegated findings in lungs and other organs suggesting vascular dysfunction. Histopathology. 2020;77(2):198-209.

21. Wang J, Saguner AM, An J, Ning Y, Yan Y, Li G. Dysfunctional coagulation in COVID-19: from cell to bedside. Adv Ther. 2020;37(7):3033-3039.

22. Freaney PM, Shah SJ, Khan SS. COVID-19 and heart failure with preserved ejection fraction. JAMA. 2020;324(15):1499-1500.

23. Mehta P, McAuley DF, Brown M, Sanchez E, Tattersall RS, Manson JJ, Hlh Across Speciality Collaboration UK. COVID-19: consider cytokine storm syndromes and immunosuppression. Lancet. 2020;395(10229):1033-1034.

24. Ruan Q, Yang K, Wang W, Jiang L, Song J. Clinical predictors of mortality due to COVID-19 based on an analysis of data of 150 patients from Wuhan, China. Intensive Care Med. 2020;46(5):846-848.

25. Mahmudpour M, Roozbeh J, Keshavarz M, Farrokhi S, Nabipour I. COVID-19 cytokine storm: The anger of inflammation. Cytokine. 2020;133:155151.

26. Coperchini F, Chiovato L, Croce L, Magri F, Rotondi M. The cytokine storm in COVID-19: An overview of the involvement of the chemokine/chemokine-receptor system. Cytokine Growth Factor Rev. 2020;53:25-32.

27. Akhmerov A, Marban E. COVID-19 and the Heart. Circ Res. 2020;126(10):1443-1455.

28. Libby P, Luscher T. COVID-19 is, in the end, an endothelial disease. Eur Heart J. 2020;41(32):3038-3044.

29. Unudurthi SD, Luthra P, Bose RJC, McCarthy JR, Kontaridis MI. Cardiac inflammation in COVID-19: Lessons from heart failure. Life Sci. 2020;260:118482.

30. Li G, Saguner AM, An J, Ning Y, Day JD, Ding L, Waintraub $\mathrm{X}$, et al. Cardiovascular disease during the COVID-19 pandemic: Think ahead, protect hearts, reduce mortality. Cardiol J. 2020;27(5):616-624.

31. Masi P, Hekimian G, Lejeune M, Chommeloux J, Desnos C, Pineton De Chambrun M, Martin-Toutain I, et al. Sys- 
temic inflammatory response syndrome is a major contributor to COVID-19-associated coagulopathy: insights from a prospective, single-center cohort study. Circulation. 2020;142(6):611-614.

32. Marchetti M. COVID-19-driven endothelial damage: complement, HIF-1, and ABL2 are potential pathways of damage and targets for cure. Ann Hematol. 2020;99(8):1701-1707.

33. Kochi AN, Tagliari AP, Forleo GB, Fassini GM, Tondo C. Cardiac and arrhythmic complications in patients with COVID-19. J Cardiovasc Electrophysiol. 2020;31(5):1003-1008.

34. Chorin E, Dai M, Shulman E, Wadhwani L, Bar-Cohen R, Barbhaiya C, Aizer A, et al. The QT interval in patients with COVID-19 treated with hydroxychloroquine and azithromycin. Nat Med. 2020;26(6):808-809.

35. Rosenberg ES, Dufort EM, Udo T, Wilberschied LA, Kumar J, Tesoriero J, Weinberg P, et al. Association of Treatment With Hydroxychloroquine or Azithromycin With In-Hospital Mortality in Patients With COVID-19 in New York State. JAMA. 2020;323(24):2493-2502.

36. Million M, Lagier JC, Gautret P, Colson P, Fournier PE, Amrane S, Hocquart M, et al. Early treatment of COVID-19 patients with hydroxychloroquine and azithromycin: A retrospective analysis of 1061 cases in Marseille, France. Travel Med Infect Dis. 2020;35:101738.

37. Singh H, Chauhan P, Kakkar AK. Hydroxychloroquine for the treatment and prophylaxis of COVID-19: The journey so far and the road ahead. Eur J Pharmacol. 2021;890:173717

38. Campbell CM, Guha A, Haque T, Neilan TG, Addison D. Repurposing immunomodulatory therapies against coronavirus disease 2019 (COVID-19) in the era of cardiac vigilance: a systematic review. J Clin Med. 2020;9(9):2935.

39. Siddamreddy S, Thotakura R, Dandu V, Kanuru S, Meegada S. Corona Virus Disease 2019 (COVID-19) Presenting as Acute ST Elevation Myocardial Infarction. Cureus. 2020;12(4):e7782.

40. Driggin E, Madhavan MV, Bikdeli B, Chuich T, Laracy J, Biondi-Zoccai G, Brown TS, et al. Cardiovascular considerations for patients, health care workers, and health systems during the COVID-19 pandemic. J Am Coll Cardiol. 2020;75(18):2352-2371.

41. European Society of Cardiology. The collateral damage of COVID-19: cardiovascular disease, the next pandemic wave. June 3, 2020. https://www.escardio.org/The-ESC/ Advocacy/Shaping-policy-and-regulation/ESC-positions/the-collateral-damage-of-covid-19-cardiovasculardisease-the-next-pandemic-wav. Updated 3 June 2020. Accessed November 11, 2020.

42. Bollmann A, Hohenstein S, Konig S, Meier-Hellmann A, Kuhlen R, Hindricks G. In-hospital mortality in heart failure in Germany during the Covid-19 pandemic. ESC Heart Fail. 2020;7(6):4416-4419.

43. De Filippo O, D'Ascenzo F, Angelini F, Bocchino PP, Conrotto F, Saglietto A, Secco GG, et al. Reduced rate of hospital admissions for ACS during COVID-19 Outbreak in Northern Italy. N Engl J Med. 2020;383(1):88-89.
44. Solomon MD, McNulty EJ, Rana JS, Leong TK, Lee C, Sung SH, Ambrosy AP, et al. The COVID-19 pandemic and the incidence of acute myocardial infarction. N Engl J Med. 2020;383(7):691-693.

45. Gori T, Lelieveld J, Munzel T. Perspective: cardiovascular disease and the Covid-19 pandemic. Basic Res Cardiol. 2020;115(3):32.

46. Fox SE, Akmatbekov A, Harbert JL, Li G, Quincy Brown J, Vander Heide RS. Pulmonary and cardiac pathology in African American patients with COVID-19: an autopsy series from New Orleans. Lancet Respir Med. 2020;8(7):681-686.

47. Kim D, Quinn J, Pinsky B, Shah NH, Brown I. Rates of co-infection between SARS-CoV-2 and other respiratory pathogens. JAMA. 2020;323(20):2085-2086.

48. Kim IC, Kim JY, Kim HA, Han S. COVID-19-related myocarditis in a 21-year-old female patient. Eur Heart J. 2020;41(19):1859.

49. Zeng JH, Liu YX, Yuan J, Wang FX, Wu WB, Li JX, Wang LF, et al. First case of COVID-19 complicated with fulminant myocarditis: a case report and insights. Infection. 2020;48(5):773-777.

50. Wang D, Hu B, Hu C, Zhu F, Liu X, Zhang J, Wang B, et al. Clinical characteristics of 138 hospitalized patients with 2019 novel coronavirus-infected pneumonia in $\mathrm{Wu}-$ han, China. JAMA. 2020;323(11):1061-1069.

51. Siripanthong B, Nazarian S, Muser D, Deo R, Santangeli P, Khanji MY, Cooper LT, Jr., et al. Recognizing COVID19-related myocarditis: The possible pathophysiology and proposed guideline for diagnosis and management. Heart Rhythm. 2020;17(9):1463-1471.

52. Babapoor-Farrokhran S, Rasekhi RT, Gill D, Babapoor S, Amanullah A. Arrhythmia in COVID-19. SN Compr Clin Med. 2020;2:1430-1435.

53. Gopinathannair R, Merchant FM, Lakkireddy DR, Etheridge SP, Feigofsky S, Han JK, Kabra R, et al. COVID-19 and cardiac arrhythmias: a global perspective on arrhythmia characteristics and management strategies. J Interv Card Electrophysiol. 2020;59(2):329-336.

54. Kir D, Mohan C, Sancassani R. Heart Brake: an unusual cardiac manifestation of COVID-19. JACC Case Rep. 2020;2(9):1252-1255.

55. Peigh G, Leya MV, Baman JR, Cantey EP, Knight BP, Flaherty JD. Novel coronavirus 19 (COVID-19) associated sinus node dysfunction: a case series. Eur Heart J Case Rep. 2020;4(FI1):1-6.

56. Chorin E, Wadhwani L, Magnani S, Dai M, Shulman E, Nadeau-Routhier C, Knotts R, et al. QT interval prolongation and torsade de pointes in patients with COVID-19 treated with hydroxychloroquine/azithromycin. Heart Rhythm. 2020;17(9):1425-1433.

57. Guo T, Fan Y, Chen M, Wu X, Zhang L, He T, Wang H, et al. Cardiovascular implications of fatal outcomes of patients with coronavirus disease 2019 (COVID-19). JAMA Cardiol. 2020;5(7):811-818.

58. Mercuro NJ, Yen CF, Shim DJ, Maher TR, McCoy CM, Zimetbaum PJ, Gold HS. Risk of QT interval prolongation associated with use of hydroxychloroquine with or without concomitant azithromycin among hospitalized 
patients testing positive for coronavirus disease 2019 (COVID-19). JAMA Cardiol. 2020;5(9):1036-1041.

59. Saleh M, Gabriels J, Chang D, Soo Kim B, Mansoor A, Mahmood E, Makker P, et al. Effect of chloroquine, hydroxychloroquine, and azithromycin on the corrected QT interval in patients with SARS-CoV-2 infection. Circ Arrhythm Electrophysiol. 2020;13(6):e008662.

60. Hadi HA, Carr CS, Al Suwaidi J. Endothelial dysfunction: cardiovascular risk factors, therapy, and outcome. Vasc Health Risk Manag. 2005;1(3):183-198.

61. Zhang J, Tecson KM, McCullough PA. Endothelial dysfunction contributes to COVID-19-associated vascular inflammation and coagulopathy. Rev Cardiovasc Med. 2020;21(3):315-319.

62. Wichmann D, Sperhake JP, Lutgehetmann M, Steurer S, Edler C, Heinemann A, Heinrich F, et al. Autopsy Findings and Venous Thromboembolism in Patients With COVID-19: A Prospective Cohort Study. Ann Intern Med. 2020;173(4):268-277.

63. Klok FA, Kruip M, van der Meer NJM, Arbous MS, Gommers D, Kant KM, Kaptein FHJ, et al. Incidence of thrombotic complications in critically ill ICU patients with COVID-19. Thromb Res. 2020;191:145-147.

64. Bilaloglu S, Aphinyanaphongs Y, Jones S, Iturrate E, Hochman J, Berger JS. Thrombosis in hospitalized patients with COVID-19 in a New York City Health System. JAMA. 2020;324(8):799-801.

65. Mosleh W, Chen K, Pfau SE, Vashist A. Endotheliitis and endothelial dysfunction in patients with COVID-19: its role in thrombosis and adverse outcomes. J Clin Med. 2020;9(6):1862.

66. Teuwen LA, Geldhof V, Pasut A, Carmeliet P. COVID-19: the vasculature unleashed. Nat Rev Immunol. 2020;20(7):389-391.

67. Huertas A, Montani D, Savale L, Pichon J, Tu L, Parent F, Guignabert C, et al. Endothelial cell dysfunction: a major player in SARS-CoV-2 infection (COVID-19)? Eur Respir J. 2020;56(1):2001634.

68. Froldi G, Dorigo P. Endothelial dysfunction in Coronavirus disease 2019 (COVID-19): Gender and age influences. Med Hypotheses. 2020;144:110015.

69. Zhang J, McCullough PA, Tecson KM. Vitamin D deficiency in association with endothelial dysfunction: Implications for patients with COVID-19. Rev Cardiovasc Med. 2020;21(3):339-344.

70. Schiavone M, Gobbi C, Biondi-Zoccai G, D'Ascenzo F, Palazzuoli A, Gasperetti A, Mitacchione G, et al. Acute Coronary Syndromes and Covid-19: Exploring the Uncertainties. J Clin Med. 2020;9(6):1683.

71. Shi S, Qin M, Shen B, Cai Y, Liu T, Yang F, Gong W, et al. Association of cardiac injury with mortality in hospitalized patients with COVID-19 in Wuhan, China. JAMA Cardiol. 2020;5(7):802-810.

72. Stefanini GG, Montorfano M, Trabattoni D, Andreini D, Ferrante G, Ancona M, Metra M, et al. ST-elevation myocardial infarction in patients with COVID-19: clinical and angiographic outcomes. Circulation. 2020;141(25):21132116.

73. Knight DS, Kotecha T, Razvi Y, Chacko L, Brown JT,
Jeetley PS, Goldring J, et al. COVID-19: myocardial injury in survivors. Circulation. 2020;142(11):1120-1122.

74. Guagliumi G, Sonzogni A, Pescetelli I, Pellegrini D, Finn AV. Microthrombi and ST-segment-elevation myocardial infarction in COVID-19. Circulation. 2020;142(8):804809.

75. Shirazi S, Mami S, Mohtadi N, Ghaysouri A, Tavan H, Nazari A, Kokhazadeh T, et al. Sudden cardiac death in COVID-19 patients, a report of three cases. Future Cardiol. 2021;17(1):113-118.

76. Mazzanti A, Briani M, Kukavica D, Bulian F, Marelli S, Trancuccio A, Monteforte N, et al. Association of Hydroxychloroquine With QTc Interval in Patients With COVID-19. Circulation. 2020;142(5):513-515.

77. Long B, Brady WJ, Koyfman A, Gottlieb M. Cardiovascular complications in COVID-19. Am J Emerg Med. 2020;38(7):1504-1507.

78. Chen T, Wu D, Chen H, Yan W, Yang D, Chen G, Ma $\mathrm{K}$, et al. Clinical characteristics of 113 deceased patients with coronavirus disease 2019: retrospective study. BMJ. 2020;368:m1091.

79. Zhou F, Yu T, Du R, Fan G, Liu Y, Liu Z, Xiang J, et al. Clinical course and risk factors for mortality of adult inpatients with COVID-19 in Wuhan, China: a retrospective cohort study. Lancet. 2020;395(10229):1054-1062.

80. Argulian E, Sud K, Vogel B, Bohra C, Garg VP, Talebi S, Lerakis $\mathrm{S}$, et al. Right ventricular dilation in hospitalized patients with COVID-19 infection. JACC Cardiovasc Imaging. 2020;13(11):2459-2461.

81. Bader F, Manla Y, Atallah B, Starling RC. Heart failure and COVID-19. Heart Fail Rev. 2021;26(1):1-10.

82. Salvatici M, Barbara B, Cioffi SMG, Morenghi E, Leone FP, Maura F, et al. Association between cardiac troponin I and mortality in patients with COVID-19. Biomarkers. 2020;25(8):634-640.

83. Goshua G, Pine AB, Meizlish ML, Chang CH, Zhang H, Bahel P, Baluha A, et al. Endotheliopathy in COVID-19-associated coagulopathy: evidence from a single-centre, cross-sectional study. Lancet Haematol. 2020;7(8):e575-e582.

84. Schott JP, Mertens AN, Bloomingdale R, O'Connell TF, Gallagher MJ, Dixon S, Abbas AE. Transthoracic echocardiographic findings in patients admitted with SARSCoV-2 infection. Echocardiography. 2020;37(10):15511556.

85. Kirkpatrick JN, Mitchell C, Taub C, Kort S, Hung J, Swaminathan M. ASE statement on protection of patients and echocardiography service providers during the 2019 novel coronavirus outbreak: endorsed by the American College of Cardiology. J Am Coll Cardiol. 2020;75(24):3078-3084.

86. Cau R, Bassareo P, Saba L. Cardiac Involvement in COVID-19-Assessment with Echocardiography and Cardiac Magnetic Resonance Imaging. SN Compr Clin Med. 2020:2:845-851.

87. Kociol RD, Cooper LT, Fang JC, Moslehi JJ, Pang PS, Sabe MA, Shah RV, et al. Recognition and Initial Management of Fulminant Myocarditis: A Scientific Statement From the American Heart Association. Circulation. 2020;141(6):e69-e92. 
88. Mahmud E, Dauerman HL, Welt FGP, Messenger JC, Rao SV, Grines C, Mattu A, et al. Management of acute myocardial infarction during the COVID-19 pandemic: a position statement from the Society for Cardiovascular Angiography and Interventions (SCAI), the American College of Cardiology (ACC), and the American College of Emergency Physicians (ACEP). J Am Coll Cardiol. 2020;76(11):1375-1384.

89. Hamadeh A, Aldujeli A, Briedis K, Tecson KM, SanzSanchez J, Al Dujeili M, Al-Obeidi A, et al. Characteristics and outcomes in patients presenting with COVID-19 and ST-segment elevation myocardial infarction. Am J Cardiol. 2020;131:1-6.

90. Beigel JH, Tomashek KM, Dodd LE, Mehta AK, Zingman BS, Kalil AC, Hohmann E, et al. Remdesivir for the Treatment of Covid-19 - Final Report. N Engl J Med. 2020;383(19):1813-1826.

91. Food and Drug Administration, US Department of Health and Human Services. FDA News Release. Coronavirus (COVID-19) update: FDA authorizes monoclonal antibodies for treatment of COVID-19. November 21, 2020. https://www.fda.gov/news-events/press-announcements/ coronavirus-covid-19-update-fda-authorizes-monoclonal-antibodies-treatment-covid-19.

92. DiNicolantonio JJ, Barroso J, McCarty M. Ivermectin may be a clinically useful anti-inflammatory agent for late-stage COVID-19. Open Heart. 2020;7(2):e001350.

93. Padhy BM, Mohanty RR, Das S, Meher BR. Therapeutic potential of ivermectin as add on treatment in COVID 19:
A systematic review and meta-analysis. J Pharm Pharm Sci. 2020;23:462-469.

94. Zhao M. Cytokine storm and immunomodulatory therapy in COVID-19: Role of chloroquine and antiIL-6 monoclonal antibodies. Int J Antimicrob Agents. 2020;55(6):105982.

95. RECOVERY Collaborative Group, Horby P, Lim WS, Emberson JR, Mafham M, Bell JL, Linsell L, et al. Dexamethasone in hospitalized patients with COVID-19 - preliminary report. N Engl J Med. 2020.

96. Chow JH, Khanna AK, Kethireddy S, Yamane D, Levine A, Jackson AM, McCurdy MT, et al. Aspirin use is associated with decreased mechanical ventilation, ICU admission, and in-hospital mortality in hospitalized patients with COVID-19. Anesth Analg. 2020.

97. Wu Q, Zhou L, Sun X, Yan Z, Hu C, Wu J, Xu L, et al. Altered Lipid Metabolism in Recovered SARS Patients Twelve Years after Infection. Sci Rep. 2017;7(1):9110.

98. Ledford H, Cyranoski D, Van Noorden R. The UK has approved a COVID vaccine - here's what scientists now want to know. Nature. 2020;588(7837):205-206.

99. Food and Drug Administration, US Department of Health and Human Services. Pfizer-BioNTech COVID-19 vaccine. December 11, 2020. https://www.fda.gov/emergency-preparedness-and-response/coronavirus-disease2019-covid-19/pfizer-biontech-covid-19-vaccine.

100. Callaway E. COVID vaccine excitement builds as Moderna reports third positive result. Nature. 2020;587(7834):337338. 